mapping supports a diverse range of learning and comprehension tasks. The hypothesis is therefore a challenging one, though not yet proven. But perhaps I have gone further than McAleese is claiming. I am certainly happy with his view of auto-monitoring as a "learning arena' in which the learner can be aware of and manipulate learning activities.

\title{
References
}

Hammond, N.V. (1993), 'Learning with hypertext: problems, principles and prospects', in McKnight, C., Dillon, A. and Richardson, J. (eds), Hypertext: A Psychological Perspective, Chichester, Ellis Horwood.

-McKendree, J. and Reader, W. (1994). "The "homeopathic fallacy" in hypertext: misconceptions of psychology in design of computer courseware', ALT News (in press).

Trapp, A.L., Reader, W. and Hammond, N.V. (1992), 'Tools for knowledge mapping: a framework for understanding' in Brusilovsky, P. and Stefanuk; V. (eds), East-West Conference on Emerging Technologies in Education, Moscow, April.

\section{Concept mapping and other formalisms as Mindtools for representing knowledge}

\author{
David H. Jonassen* and Rose M. Marra** \\ ${ }^{\star}$ Pennsylvania State University and ** AT\&T, USA
}

\section{Introduction}

We seek to provide an alternative theoretical perspective on concept mapping (a formalism for representing structural knowledge) to that provided by Ray McAleese in this issue of ALT-J (auto-monitoring). We begin with an overview of concept maps as a means of describing a learner's knowledge constructs, and then discuss a broader class of tools, Mindtools, of which concept maps are a member. We proceed by defining Mindtools as formalisms for representing knowledge, and further elaborate on concept maps as a formalism for representing a particular kind of knowledge: structural knowledge. We then address McAleese's use of the term auto-monitoring and some of the steps in his model of concept maps. Finally, we describe some limitations of concept mapping as a formalism and as a cognitive learning strategy.

Concept maps, both in how they are used and theoretically based, touch some of the central elements of instructional technology and learning. Concept maps address the critical issue of how we organize our knowledge structures within and between domains. They, in some sense, model how we represent and apply knowledge. Assuming that instructional technology, as a field, has a goal of being able systematically to make instruction more effective, then tools 
such as concept maps that allow us to see and understand what the learner is starting with seem critical. Concept maps may fall into a larger category of tools, Mindtools, that enhance our understanding of how learners organize and use knowledge.

\section{Mindtools}

Mindtools are computer-based tools and learning environments that have been adapted or developed to support critical thinking in the form of knowledge representation (Jonassen, in press). These tools include (but are not necessarily limited to) databases, microworlds and learning environments, spreadsheets, semantic networks and concept maps, expert systems, multimedia construction, computer-mediated communication, and programming languages. Mindtools, otherwise known as cognitive tools (Kommers et al 1992), or 'content-free courseware' as McAleese refers to them, are generalizable computer tools that are intended to engage and facilitate cognitive and meta-cognitive processing - hence cognitive tools. Derry (1990) has defined cognitive tools as both mental and computational devices that support, guide, and extend the thinking processes of their users. They are knowledge-construction and facilitation tools that can be applied to a variety of subject-matter domains. We believe that students cannot use these tools without thinking deeply about the content that they are learning.

Cognitive tools are an example of learning with information processing technologies as opposed to learning from them (Salomon et al 1991). Learning with technologies amplifies the learner's cognitive processes while using those technologies. Computer-based cognitive tools and environments are, in effect, cognitive amplification tools. Environments that employ cognitive tools distribute cognition to the person plus their 'surround' - the physical and social resources available to them (Perkins 1993). This is perhaps the most powerful rationale for Mindtools. If we consider the learner and the computer as parts of a total learning system, our goal should be to distribute the cognitive responsibility appropriately to the different parts of the system. Rather than requiring learners to store and retrieve information, which is something that computers do much better, relegate those responsibilities to the computer and require the learners to identify patterns, develop organizational schemes, and synthesize information, which are things that humans do much better than computers. Mindtools more appropriately distribute the cognitive responsibilities to the appropriate parts of the learning system.

Mindtools are not intended necessarily to reduce information processing, that is, make learning easier, as has been the goal of instructional design and most instructional technologies, but rather to engage learners in more effective processes of learning. Nor are they 'fingertip' tools (Perkins 1993) that learners use naturally, effortlessly, and effectively. Rather, Mindtools provide an environment and vehicle that often require learners to think harder about the subject-matter domain being studied while generating thoughts that would be impossible without the tool. They are cognitive reflection and amplification tools that help leamers to construct their own realities using the constructs and processes in the environment on a new content domain. McAleese's description of a learner engaged in a 'cognitively active process in an environment that supports the manipulation of knowledge artefacts' could in fact be described as a learner who is engaged with some sort of a Mindtool - in this case, one that supports concept mapping. The tool allows the learner to represent and explicitly manipulate knowledge formalisms and thus 'amplifies' or makes concrete what would otherwise be abstract and difficult to grasp. 


\section{Mindtools as representational formalisms}

Mindtools consist of different formalisms for representing knowledge; that is, they use different schemes that can be used to represent content or personal knowledge. Expository text is the most common formalism that we use to capture and represent what we know or have learned. However, expository prose constrains the learner's representation. Writing is a complex activity whose syntax and multiple text-structures entail a great deal of cognitive overhead.

Mindtools provide different, more constrained formalisms for representing personal knowledge. Mindtools, like text, require that learners represent domain knowledge in a knowledge base with formal, constrained statements of relationships between the ideas in the knowledge base. However, the relationships and fundamental structures that are generated by each formalism vary. There are many classes of Mindtools. Each class engages different critical thinking skills (Jonassen, in press) and involves different syntax and representational formalisms. Since this issue focuses on concept mapping, we shall next treat it in greater depth.

\section{Mapping structural knowledge via concept maps}

Concept mapping is a formalism for representing structural knowledge (Jonassen et al 1993). Structural knowledge is the knowledge of how concepts within a domain are interrelated (Diekhoff 1983). If schemas are defined by their interrelationships to other schemas, then explicit awareness of those interrelationships and the ability to explicate those relationships are essential for higher-order procedural knowledge. Declarative knowledge is not a sufficient prerequisite for procedural knowledge. In order to acquire procedural knowledge, it is necessary to understand the propositional relationships between the entities involved in both the procedural and declarative knowledge structures.

Structural knowledge provides that link between procedural and declarative knowledge. Structural knowledge, the understanding of these relationships, enables learners to form the connections they need in order to use scripts or complex schemas: that is, the ability actually to apply what they know. Structural knowledge has been conceived and described by. several different authors (Preece 1976; Tennyson and Cocciarella 1986), but the underlying assumption of all descriptions of structural knowledge is that meaning for any concept or construct is implicit in the pattern of relationships to other concept or constructs.

Concept mapping, whether accomplished with paper and pencil or using sophisticated semantic networking tools such as SemNet, Learning Tool, TextVision, or CMap, are means for representing cognitive structures (Jonassen 1987), ideas and their complex interrelationships (schemata) in memory that provide meaning to the language used to transmit those ideas.

Schema theory and semantic networking theory are the theoretical bases for concept mapping. These theories are based on schemas, which are arranged in networks (schemata) of interrelated concepts. These networks are known as semantic networks. Perhaps the best-known conceptualization of a semantic network is active structural networks (Quillian 1968), structures that are composed of nodes (the equivalent of schemas) and ordered, typed relationships or links connecting them. The nodes are token instances of concepts or propositions, and the links described the propositional relationship between them. As such, concept maps and semantic networks describe a person's knowledge of the interrelationships between ideas 
(Jonassen et al 1993). In addition to the tools mentioned above (SemNet, etc.) that allow learners to create their own concept maps, there are other tools designed to create concept maps from similarity ratings between concept pairs in a particular domain.

These raw similarity ratings from the learners are algorithmically transformed (via an algorithm such as Pathfinder Net - Schvaneveldt et al 1985) into a concept-map form of knowledge representation. This representation can be evaluated via some criteria, such as whether it can accurately classify members of dissimilar classes (for example, expert and novice). Pathfinder nets is another concept-mapping tool used to represent structural knowledge.

\section{Response to McAleese: auto-monitoring and internal negotiations}

So far, our commentary has centred on our view of concept 'maps and how they fit with the concept of Mindtools and structural knowledge. This section discusses a couple of points from McAleese's concept-mapping rationale. We shall start by looking at the idea of auto-monitoring and how it relates to some previously addressed ideas, and then address McAleese's stages of concept modelling from a more constructivistic perspective.

\section{Structural knowledge, meta-cognition and auto-monitoring}

Perhaps the difference between these terms is just a matter of semantics, but even if that is the case, it is worth discussing, since meaning (semantics) is the essence of the topic we are discussing. We consider McAleese's use of the term auto-monitoring. We propose that there is very little difference between McAleese's term and other ideas - namely, what we have previously described as structural knowledge and what is universally known as meta-cognition. The rationale for our concern is a growing need for parsimony in our communications.

McAleese defines auto-monitoring as a technique 'of personal or group knowledge presentation and re-presentation', and further indicates that these techniques are often supported by various computer applications that graphically represent knowledge structures. He further acknowledges that the 'main problem' with auto-monitoring is its universality; that is, McAleese himself admits the influence of the concepts of meta-cognition and reflective practice on auto-monitoring. We agree with these influences, and would prefer to say that the distinction between these concepts and auto-monitoring is perhaps only in the application of the term auto-monitoring to the meta-cognitive process that occurs as a learner self-negotiates while creating a concept map.

McAleese argues that auto-monitoring is distinct from meta-cognition because auto-monitoring not only is aware of the cognitive processes, but also is in charge of creating them. We reply that without the awareness of the creation process and the knowledge structures (declarative and procedural) on which the creation is based, the creation itself would be impossible. In other words, the meta-cognitive controls that allow leamers to look inward and understand their own knowledge structures is a necessary step for the subsequent instantiation of these structures into a cognitive map. This is supported by the common distinction in the metacognition literature between executive control processes and comprehension monitoring.

Further, we would argue that what learners must actually be aware of is not only their declarative and procedural knowledge structures, but the structural knowledge structures that link and provide the meaning necessary to apply the previous two. 


\section{Commitment, checking and confirming: a constructivistic perspective}

McAleese describes a series of stages in which learners engage when creating a concept map. Once concepts and nodes have been created, the learner engages in a process of linking nodes and describing the relationship between nodes, and then a re-scrutinization of those nodes and links (checking) to make sure they agree with their representations and resolving any conflicts discovered in labelling links (resolution). From a constructivist perspective, this process of learners attempting to resolve their own conflicts about how to represent and label knowledge is referred to as internal negotiation (Jonassen $e t$ al, in press). Just as meaning is derived and socially negotiated between individuals from a constructivist perspective, so individuals must do the same with themselves. We engage in this internal negotiation activity almost constantly, and often without explicitly realizing it as we process new stimuli and link them to existing concepts and knowledge structures. Perhaps the greatest strength of concept-mapping tools is that they make learners deal explicitly and consciously with what is normally an implicit activity. And further, explicitly instantiating this normally implicit/internal process may be why it is difficult for some learners to commit to a particular concept map. (Tools such as Pathfinder nets that create a concept map from similarity ratings avoid some of the difficulty of the learner having to sort out all of the relationships for themselves).

McAleese describes the confirming stage as where the learner 'agrees' that the representation is what was intended and further elaborates on the significance of the 'commitment' process. We wish to agree with McAleese's position that total commitment to a learner's representation may not occur due to cognitive dissonance and the tension between describing a concept relationship in one of several similar fashions. Further, we emphasize that it seems very likely that a map a learner creates in one sitting (and thought they were 'finished' with), may look very incomplete or even incorrect when revisited. This seems perfectly reasonable, given the fluid nature of an individual's knowledge structures. In different contexts or at different times, an individual's representation will be very different.

Once again, from a constructivistic perspective, existing knowledge structures are continually being modified as we sense and process and meld new ideas to existing schemas and schemata. If this is the case, then a learner's conception of relationships between various concepts will undoubtedly change, thus making total commitment to a concept map over a long period of time seem rather unlikely. Like McAleese we would agree this is a good thing in that if learners (and instructors) are aware that their views of the world may legitimately change and grow, then more may be gained by revisiting previous concept maps.

\section{Limitations of concept maps and semantic networks}

It is easy to become enamoured of representational tools like concept mapping and semantic networking. They are powerful tools, but they are easily attributed too much power. We now discuss a few cautionary notes.

\section{Reification of representations}

Semantic network theory is an example of a symbolic processing model of cognition. This traditional view of learning assumes that human memory consists of stored facts and procedures. Clancy (1992) challenges the 'identity hypothesis' of these symbolic representation models, claiming that semantic-network theorists see nets as isomorphic representations of what is stored in memory. That is, memory structures (and by inference, structural knowledge) 
literally exist as representations in the mind - that they represent not only behaviour but also internal representational structures and mental processes. Concept maps and semantic networks can be too readily thought to reify the structures of the mind. This implies that the semantic store of information in humans can be cognitively mapped and literally searched just as a computer searches its memory stores. These assumptions impute far too much reality to concept maps. They are not truly maps of the mind, but rather representations of what we think is in the mind. In truth, they are not even really good at that for the following reasons.

\section{Ephemerality, dynamics, and context dependence of semantic networks}

Connectionist models, the alternatives to symbolic representation models, believe that memory is distributed in different memory locations. They see structural knowledge as neural networks that encode memories in multiple locations - that memory is not static as symbolic representations assume. Memory changes according to the context in which the events occurred or the context in which ideas are being recalled. Structural knowledge is dynamic. What is recalled depends on the context, the need, and a number of other variables.

Structural knowledge is therefore ephemeral. It is not the same over time. Yet many people take concepts maps as literal, static, and immutable representations of what is known. Concept mapping tools would need to enable minute-by-minute, context-by-context changes in the concepts, relationships and structures that are represented in it in order more accurately to represent knowledge.

\section{Complexity of internal semantic networks and simplicity of their representations}

The propositional networks in the mind, in whatever form they really exist, are far more complex than anything that can be represented in a concept map. Conceptually, all the constructs that we know are interrelated and multiply, encoded in rich, very redundant networks of ideas. Concepts are not just linked to one another in one way. Two concepts may be linked in multiple ways. These networks are multidimensional, yet $n$-dimensionality is a concept that is extremely difficult to grasp and even harder to represent in two or threedimensional space. At best, concept maps are distillations of what we know.

\section{Conclusion}

In this short contribution, we have argued that:

- the main strength of concept maps is making explicit what is normally implicit;

- we agree with much of what McAleese proposes but do not see auto-monitoring as different from meta-cognition and structural knowledge;

- from a constructivist perspective, the concept map creation process supports the rele of internal knowledge negotiation;

- concept maps should be interpreted cautiously and used only as an ephemeral knowledge-representation tool for internally and socially negotiating the knowledge construction process. 


\section{References}

Clancy, W.J. (1992). 'Representations of knowing: in defense of cognitive apprenticeship.' Journal of Artifical Intelligence in Education, 3 139-168.

Derry, S.J. (1990), 'Flexible cognitive tools for problem solving instruction', paper presented at the annual meeting of the American Educational Research Association, Boston MA, April 16-20.

Diekhoff, G.M. (1983), 'Relationship judgments in the evaluation of structural understanding', Journal of Educational Psychology, 75, 227-233.

Jonassen, D.H., Beissner, K. and Yacci, M.A. (1993), Structural Knowledge: Techniques for Conveying, Assessing, and Acquiring Structural Knowledge, Hillsdale NJ, Lawrence Erlbaum.

Jonassen, D.H., Davidson, M.E., Campbell, J.P., Haag, B.B. and Hettinger, G.A. (in press), 'Evaluating learning from constructivist learning environments', Training Research Journal.

Jonassen, D.H. (1987), 'Assessing cognitive structure: verifying a method using pattern notes', Journal of Research and Development in Education, 20 (3), 1-14.

Jonassen, D.H. (in press), Mindtools for Schools, New York, Macmillan.

Kommers, P.A.M., Jonassen, D.H., \& Mayes, T. (1992), Cognitive Tools for Learning, Berlin, Springer Verlag.

Perkins, D.N. (1993), 'PERSON PLUS: a distributed view of thinking and learning' in Salomon, G. (ed), Distributed Cognitions. Cambridge University Press.

Preece, P.F.W. (1976), 'Mapping cognitive structure: a comparison of methods', Journal of Educational Psychology, 68, 1-8.

Quillian, M.R. (1968), 'Semantic memory' in Minsky, M. (ed), Semantic Information Processing, Cambridge MA, MIT Press.

Salomon, G., Perkins, D.N. and Globerson, T. (1991), 'Partners in cognition: extending human intelligence with intelligent technologies', Educational Researcher, 20, 3, 2-9.

Schvaneveldt, R.W., Durso, F.T., Goldsmith, T.E., Breen, T.J., Cooke, N.M., Tucker, R.G. and DeMaio, J.C. (1985), 'Measuring the structure of expertise, International Journal of ManMachine Studies, 23, 699-728.

Tennyson, R.D. and Cocciarella, M.J. (1986), 'An empirically based instructional design theory for teaching concepts', Review of Educational Research, 56, 40-71. 